\title{
AVALIAÇÃO DA EFETIVIDADE DE ÁREAS PROTEGIDAS: CONCEITOS, MÉTODOS E DESAFIOS
}

\section{Yata Anderson Gonzaga Masullo*} Instituto Maranhense de Estudos Socioeconômicos e Cartográficos

\section{Helen Gurgel ${ }^{\star \star}$}

Universidade de Brasília

\section{Anne-Elisabeth Laques ${ }^{* * *}$}

Institut de Recherche pour le Développement

Resumo: A criação das áreas protegidas é considerada como a principal estratégia para preservação e conservação do patrimônio natural e cultural do planeta (Diegues, 2000). Entretanto, Hockings et al. (2006) afirmam que, para alcançar tais objetivos, faz-se necessário efetivar e avançar com o gerenciamento e a administração dessas áreas protegidas. Nesse cenário surgem metodologias e procedimentos técnicos que visam subsidiar o planejamento e a tomada de decisão, ganhando cada vez mais espaço na implementação e na efetivação de políticas públicas, relacionadas à temática socioeconômica e ambiental. Desse modo, o presente estudo analisa as complexidades e os desafios de se implementar áreas protegidas, bem como se busca debater sobre as principais formas e definições conceituais da efetividade das APs e diferentes abordagens sobre a temática. Visa-se dessa forma avançar nas discussões metodológicas de avaliação do nível de efetividade e vulnerabilidade socioambiental desses territórios protegidos, de modo que seja possível analisar a inter-relação das variáveis de forma agregada e transversal.

Palavras-chave: Áreas protegidas. Efetividade. Planejamento e gestão.

\section{EVALUATION OF THE EFFECTIVENESS OF PROTECTED AREAS: CONCEPTS, METHODS AND CHALLENGES}

Abstract: The creation of protected areas is considered as the main strategy for preservation and conservation of the natural and cultural heritage of the planet (Diegues, 2000). However, Hockings et al. (2006) state that in order to achieve these objectives, it is necessary to carry out and advance the management and administration of these protected areas. In this scenario, there are technical methodologies and procedures that aim to subsidize planning and decision making, gaining more and more space in the implementation and implementation of public policies related to socioeconomic and environmental issues. Thus, the present study analyzes the complexities and challenges of implementing protected areas, as well as discusses the main forms and conceptual definitions of the effectiveness of PAs and different approaches on the theme. It is intended to advance in the methodological discussions to assess the level of effectiveness and social and environmental vulnerability of these protected territories, so that it is possible to analyze the interrelationship of variables in an aggregated and transversal way. Keywords: Protected areas. Effectiveness. Planning and management.

\section{ÉVALUATION DE L'EFFICACITÉ DES AIRES PROTÉGÉES: CONCEPTS, METHODES ET DÉFIS}

Reseumé: La création d'aires protégées est considérée comme la principale stratégie de préservation et de conservation du patrimoine naturel et culturel de la planète (Diegues, 2000). Cependant, Hockings et al. (2006) indiquent que pour atteindre ces objectifs, il est nécessaire de mener à bien la gestion et l'administration de ces aires protégées. Dans ce scénario, des méthodologies et des procédures techniques visent à subventionner la planification et la prise de décision, en donnant de plus en plus de place à la mise en œuvre et à la mise en œuvre des politiques publiques relatives aux questions socioéconomiques et environnementales. Ainsi, la présente étude analyse les complexités et les défis de la mise en œuvre des aires protégées, ainsi que les principales formes et définitions conceptuelles de l'efficacité des AP et les différentes approches sur le thème. Il est prévu d'avancer dans les discussions méthodologiques pour évaluer le niveau d'efficacité et la vulnérabilité sociale et environnementale de ces territoires protégés, afin qu'il soit possible d'analyser la corrélation des variables de manière agrégée et transversale. Mots-clés: Aires protégées. L'efficacité. Planification et gestion.

*Doutor em Geografia. Pesquisador do Instituto Maranhense de Estudos Socioeconômicos e Cartográficos - IMESC. Endereço: Av. do Vale, Ed. Zircônio, $1^{\circ}$ andar, Renascença II, CEP. 65075-820, São Luís/MA, Brasil. ORCID: https://orcid.org/0000-0001-5861-752X. E-mail: yanderson3@hotmail.com.

**Doutora em Geografia. Professora adjunta da Universidade de Brasília - UNB. Endereço: Instituto de Ciências Humanas - IH, Departamento de Geografia - GEA CEP 70910 900, Brasilia/DF, Brasil. ORCID: https://orcid.org/0000-0002-4250-6742. E-mail: helengurgel@unb.br.

${ }^{*}$ Pesquisadora do Institut de Recherche pour le Développement (IRD), UMR ESPACE-DEV. Endereço: 34093 Montpellier cedex 5, França. ORCID: https://orcid.org/0000-00034469-270X. E-mail: anne-elisabeth.laques@ird.fr. 


\section{Introdução}

As áreas protegidas - APs têm sido uma ferramenta de gestão bem-sucedida para preservar a biodiversidade, pois sem a existência destes territórios a perda global de biodiversidade seria considerávelmente ampliada (UNEP; WCMC; IUCN; NGS, 2018). Essa perspectiva é reconhecida por várias instituições e políticas internacionais, incluindo a Agenda 2030 para Desenvolvimento Sustentável, a Convenção sobre Diversidade Biológica (CDB), a Convenção de Zonas Úmidas de Importância Internacional e Convenção Ramsar.

Considerando a necessidade de se viabilizar a conservação e a proteção dos recursos naturais e culturais, o percentual das APs no mundo devese alcançar $17 \%$ das áreas terrestres e de águas continentais e $10 \%$ de áreas marinhas e costeiras até 2020, conforme as Metas de Biodiversidade de Aichi (UNEP; WCMC; UICN; NGS, 2018). Em 2018, segundo o WDPA - World Database on Protect Areas (Banco de Dados Mundial de Áreas Protegidas), 238.563 territórios são registrados como APs, contribuindo com a conservação de aproximadamente 20 milhões de $\mathrm{km}^{2}$ ou $14,9 \%$ da superfície terrestre do planeta. Contudo verifica-se que as APs terrestres registraram redução de 0,5 pontos percentuais entre os anos de 2014 e 2018 (Juffe-Bignoli et al., 2014; UNEP; WCMC; UICN; NGS, 2018)

Diante deste contexto questiona-se quais medidas são prioritárias para otimização da gestão de territórios protegidos ou como o contexto local influencia na proteção e na conservação dos recursos naturais e culturais. E, principalmente, como quantificar e repassar informações de forma sintética aos gestores e à sociedade como um todo.

Essas questões ganham ainda mais importância quando se observa que um número considerável de APs no mundo se encontra em situação denominada por Terborgh e Schaik (2002) como "parques de papel", se referindo às APs que foram apenas instituídas e não efetivadas. Para ampliar as ações que alterem esse panorama, é necessário aumentar a coleta e a análise de dados que permita a construção de metodologias, com o intuito de desenvolver interpretações realísticas dos fenômenos, bem como otimizar o planejamento estratégico e a gestão integrada.

Assim, pesquisas para a construção de indicadores sintéticos são importantes para a formulação de conceitos e metodologias (PADOVAN, 2003; ERVIN, 2003; STOLTON et al., 2007; WWF, 1999; MARINELLI, 2011; MASULLO et al., 2018a) que se aproximem da realidade complexa de países como o Brasil. No atual cenário, o planejamento e a gestão das APs se complexifica, influenciados por aspectos econômicos, sociais, culturais e políticos, originários da relação conflituosa entre a sociedade e a natureza.

Nesse sentido, o presente estudo objetiva analisar o contexto atual e a complexidade de se efetivar APs, bem como avaliar diferentes metodologias desenvolvidas e aplicadas em diversos países e posteriormente adaptadas à realidade da América Latina e brasileira.

0 estudo primeiramente analisa as complexidades e os desafios de se implementar as APs como uma estratégia efetiva de proteção e preservação do patrimônio natural e cultural. Para tanto, destacam-se as principais formas e definições conceituais da efetividade das APs e diferentes abordagens sobre a temática. Num segundo momento, promove-se um debate a partir do ensaio teórico sobre os conceitos, aplicações e desafios dos indicadores e índices sintéticos ligados ao planejamento e à gestão de áreas protegidas. Por fim, reflete-se sobre os avanços e desafios para a institucionalização e a efetivação desses territórios protegidos.

Planejamento, gestão e efetividade das áreas protegidas

Acredita-se que as APs são a espinha dorsal da conservação da biodiversidade, bem como oferecem uma gama de outros benefícios sociais, econômicos e ambientais (LEVERINGTON et al., 2010). Seguindo esse pressuposto, entende-se o porquê de a sociedade investir recursos na criação e na gestão desses territórios protegidos.

Contudo faz-senecessário avaliar atéque ponto essas APs realmente protegem seus valores e proporcionam benefícios à comunidade (STOLL-KLEEMANN E JOB, 2008). Entende-se que a simples institucionalização e o estabelecimento de regras e normas não garante um sistema capaz de alcançar efetividade desses territórios.

Refletindo sobre a temática Hockings et al. (2015), entende-se como uma AP efetiva quando esta possui capacidade e competências particulares que permitam a essa AP cumprir satisfatoriamente com as funções para as quais foi criada. Geldmann et al. (2015) complementam esse entendimento afirmando que uma AP pode ser considerada como efetiva quando alcança seus objetivos em relação à manutenção da integridade e da resiliência, de modo a garantir a representatividade e a viabilidade de todos os níveis de organização da biodiversidade. 
Para Silva (2016) a efetividade na gestão desses territórios refere-se ao êxito da AP em relação à conservação, à manutenção dos valores da biodiversidade e à sustentabilidade no uso dos recursos naturais. Essa capacidade, segundo a autora, é comumente avaliada através de métodos que por muitas vezes sobrepõem conceitos como efetividade ,eficiência e eficácia. Originam-se a partir destes princípios técnicas de avaliação que pressupõem a existência de níveis diferenciados de qualidade de gestão e de implementação das APs (GIDSICKI, 2013).

Infere-se a avaliação da efetividade das APs, o objetivo de mensurar o grau de eficiência, ou seja, o quão bem é gerenciada e qual extensão do que se pretende conservar, concentrando-se nos resultados e metas (HOCKINGS et al., 2006). Complementando Watts (2016), afirma-se que, para se avaliar a efetividade das
APs, se deve analisar a capacidade desta em executar as ações relacionadas com seus objetivos de promover a conservação e a proteção da natureza e do patrimônio cultural. Isso necessariamente envolve recursos humanos e financeiros, infraestrutura, plano de manejo e participação social ativa. Assim, as metodologias de avaliação da efetividade das APs passam a ser consideradas como pré-requisito para conservação e proteção do ambiente natural e sociocultural. Geztner (2012) demonstra que essas metodologias utilizadas atualmente são extremamente variáveis em relação a tamanho, finalidade, eficácia, infraestrutura política e jurídica, bem como financiamento, pesquisa e formas de monitoramento. Percebe-se a importância e a complexidade conceitual e metodológica que se enfrenta. Para Hockings (2006) para se ampliar a efetividade das APs deve seguir seis elementos principais (Figura 1).

Figura 1 - Elementos para efetivação de áreas protegidas.

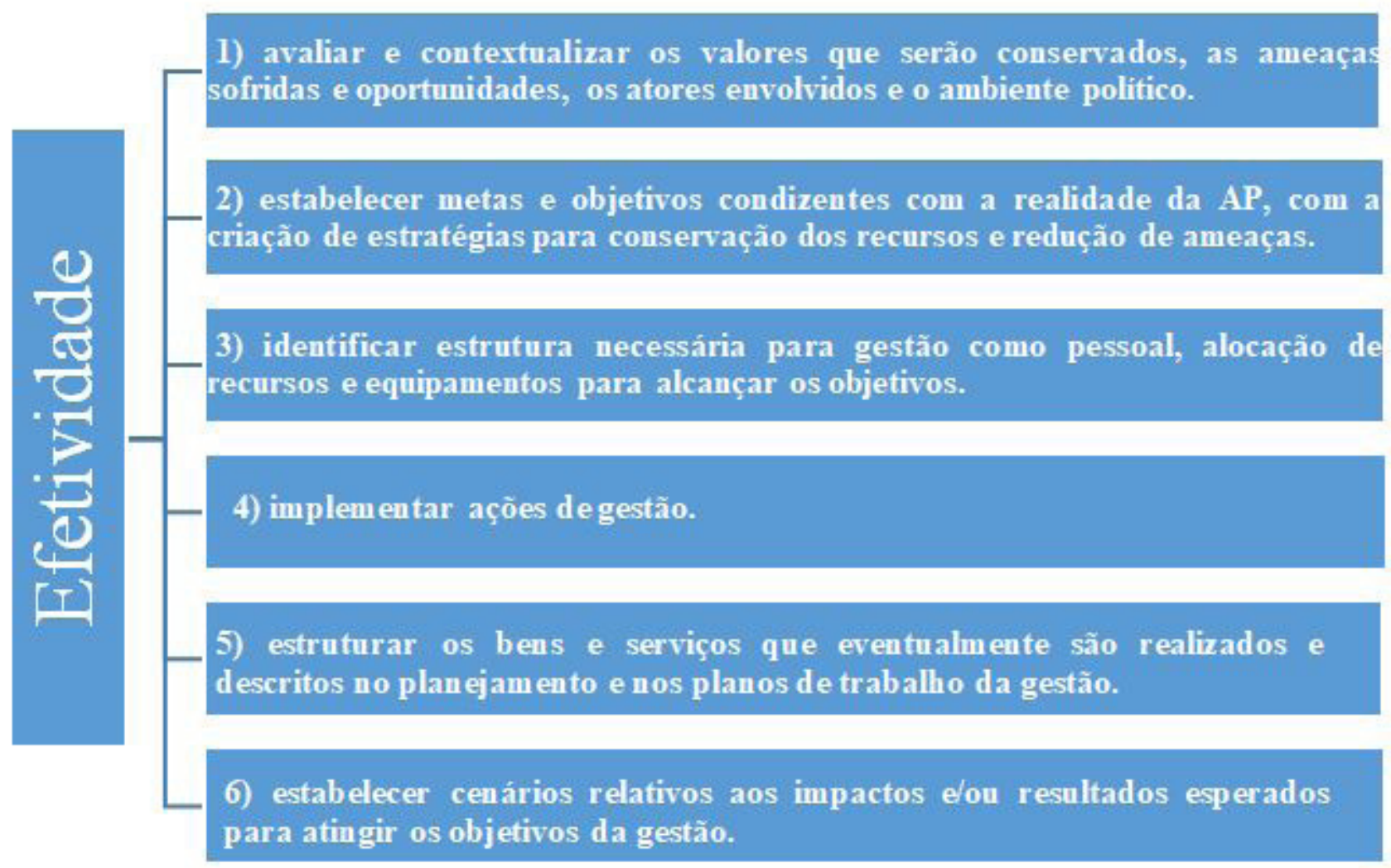

Fonte: Hockings (2006). 
Quadro 1 - Níveis de avaliação da eficácia de áreas protegidas.

\begin{tabular}{|c|c|}
\hline NIVEL & DESCRIÇÃO \\
\hline 1 & $\begin{array}{l}\text { Avalia-se o uso e a cobertura da terra e demonstra-se até que ponto a } \\
\text { biodiversidade está conservada. }\end{array}$ \\
\hline 2 & $\begin{array}{l}\text { Investiga-se as relações entre as áreas protegidas e os impactos } \\
\text { ambientais. Esses estudos fornecem informações essenciais e objetivas } \\
\text { sobre o sucesso da conservação em um nível amplo, mas têm limitações } \\
\text { significativas por depender de um conjunto limitado de indicadores. }\end{array}$ \\
\hline 3 & $\begin{array}{l}\text { Direciona-se a partir dos elementos ligados a gestão: contexto, } \\
\text { planejamento, insumos, processos e resultados, busca a coleta de dados } \\
\text { junto aos gestores e comunidade em geral, com o objetivo de auxiliar na } \\
\text { priorização de recursos e agrupamento de indicadores. }\end{array}$ \\
\hline 4 & $\begin{array}{l}\text { Avalia-se o monitoramento detalhado da condição e da tendência de } \\
\text { valores específicos da AP, tais como populações de animais, condições } \\
\text { florestais, valores culturais e impactos socioeconômicos. }\end{array}$ \\
\hline
\end{tabular}

Fonte: adaptado de Leverington et al. (2010).

Leverington et al. (2010) apontam quatro níveis diferentes e complementares para avaliação da efetividade das APs (Quadro 1).

De acordo com Schreckenberg et al., (2016) para alcançar a efetividade das APs, requer que sejam "gerenciadas de forma equitativa". Por outro lado Leverington et al. (2010), expõem que as metodologias de avaliação de efetividade de APs em geral seguem 4 abordagens (Quadro 02).

Conforme Schulze et al. (2017) entre essas abordagens metodológicas supracitadas, a mais utilizada é a Protect Area Management Effectiveness - PAME${ }^{1}$. Como exemplo de método que utiliza essa abordagem identifica-se o Rapid Assessment and Priozation of Protect Area Management - RAPPAM, com base em uma visão sistêmica essa abordagem tornou-

1A PAME visa fornecer uma avaliação de quão bem uma área protegida está sendo gerenciada: principalmente na medida em que a gestão está protegendo os valores da AP (conservação da biodiversidade, serviços ecossistêmicos e prestação de serviços culturais) (HOCKINGS et al., 2006) se uma valiosa fonte de avaliação de ameaças ${ }^{2}$ as APs. Sejam estas ameaças ambientais, socioeconômicas ou mesmo políticas, que influenciam ou impedem a efetivação das políticas públicas.

Sob essas difererentes e complementares perspectivas, observa-se que avaliar os diversos elementos e ameaças constantes nas APs permite mensurar o esforço necessário a ser despendido em sua conservação e, consequentemente, o seu nível de efetividade (JENKINS E JOPPA, 2009). No entanto, conforme Geldmann et al. (2014), independente da metodologia e da abordagem a serem utilizadas nesse processo, persistem diversas lacunas principalmente no que tange ao seu papel social. Desse modo, antes de se avançar nestas perspectivas, faz-se necessário compreender que hoje as funções das APs devem ser 
consideradas, e em parte priorizadas, no seu contexto social (MCNEELY, 2004; SCHERL, 2006; STOLLKLEEMANN, 2010; NOLTE et al., 2013).

Necessariamente, isso não depende exclusivamente do gestor da AP ou da população local, mas de uma articulação que perpassa os níveis federal, estadual e local. Nesse contexto, Franks e Schreckenberg (2016) reafirmam que a efetividade das APs passa pelo entendimento das questões socioeconômicas e sua influência sobre os processos e interações ecológicas.

Para autores como Wilkie et al. (2006) e HirschnitzGarbers e Stoll-Kleemann (2011) existe a necessidade de se avaliar o bem-estar das populações locais e no entorno, alinhando análises da dinâmica espaço-tempo do uso e da cobertura da terra, com o nível de coesão da comunidade; o desenvolvimento de benefícios às populações locais; condições socioeconômicas, políticas e institucionais; percepções e atitudes dos atores sociais envolvidos e dos órgãos gestores.

Busca-se assim compreender os riscos, as pressões e não só a aceitação e a integração da população local com o modelo de governança, mas a articulação entre os diferentes setores da sociedade (MASULLO et al., 2018b). Essa abordagem integrativa possibilita atender questões ligadas à consecução da conservação da biodiversidade, desenvolvimento comunitário, alívio da pobreza e outros objetivos interligados ao manejo (HOCKINGS et al., 2004; BERTZKY et al., 2012). Sendo assim, a influência dos seres humanos sobre a AP deve ser, portanto, considerada a partir de paradigmas mais amplos.

Percebe-se que há uma influência dos processos culturais sobre a diversidade biológica e que essa é uma dimensão a ser discutida no âmbito das práticas de proteção da natureza (CASTRO JÚNIOR et al., 2009). Os autores ressaltam que no Brasil essa realidade é ainda mais evidente, pois um grande número de unidades de conservação $-U^{3} C^{3}$ já foi estabelecido em locais com grandes diversidades socioeconômicas e espaciais.

Visualiza-se então a formação das APs como um território de múltiplas escalas, formado por um sistema socioambiental e institucional de vários níveis. 0 reconhecimento explícito dessas interações possibilita a compreensão de sua dinâmica e, por conseguinte, apresenta caminhos para ampliar a sua efetividade (GRAEME et al., 2015). Corroboram com esse modelo conceitual Cifuentes et al. (2000); Stoll-Kleemann (2010), Schaefer et al. (2015). Esses estudos supracitados registram que a existência de uma gestão intraunidade não é suficiente para se produzir resultados positivos em determinados níveis, mesmo porque existem diversos atores sociais que influenciam o nível de qualidade do gerenciamento em diferentes dimensões e escalas, possibilitando ou não a efetividade dessas APs.

Compreende-se, dessa forma, que a efetividade das APs passa a ser diretamente influenciada pelas ameaças, pressões, características territoriais e sua resiliência. Segundo Dudley et al. (2008), torna-se possível, dessa forma, identificar as APs com maior estágio de alteração na paisagem, auxiliar na priorização dos esforços e dos investimentos para a conservação, bem como monitorar a performance das metas da AP e das políticas públicas implementadas nesses territórios.

Souza (2015) ressalta que para efetivar as políticas públicas é imprescindível o desenvolvimento de intervenções transversais por parte do poder público. No caso específico da gestão das UCs, esta passa a ser compreendida como um modelo de gerenciamento que deve promover uma gestão adaptativa, articulando os componentes institucionais e os diversos agentes sociais que influenciam o planejamento e a gestão dentro da UC e na área de entorno. Essa perspectiva enquadra as UCs no Brasil em um quadro mais amplo de desenvolvimento socioespacial e regional.

A efetividade das APs passa então pela reconstrução das singularidades do território e suas múltiplas interrelações (SCHAEFER et al., 2015). O avanço contido nessa perspectiva é a tentativa de se compreender de forma integrada como os avanços sociais podem ampliar a efetividade das APs, através de ações voltadas a promoção da agricultura familiar, serviços de educação e geração de renda. Visa-se, assim, através do aumento da participação social, mitigar grande parte dos conflitos locais, além de implementar as estratégias de gestão ambiental e territorial em escalas regional e local (MASULLO et al., 2018b).

Em meio às questões apresentadas e ao desenvolvimento de técnicas e métodos relacionados à mensuração da efetividade de APs, permanece uma questão: a efetivação das APs possibilitará a ampliação da conservação ambiental e da preservação do patrimônio cultural? Uma resposta simples para a questão não parece viável, mas refletir sobre como a dinâmica territorial em diferentes escalas interage com as APs apresenta-se cada vez mais como crucial para desenvolver políticas públicas que alcancem resultados reais. 
Indicadores e índices: vantagens e limitações

Estudos que envolvem a temática em estudo agregam fenômenos interligados ao adensamento populacional, à segregação socioespacial, aos processos de exclusão social e alterações ambientais, acesso a serviços públicos, assim como ausência ou inoperância de políticas públicas eficazes (MAIOR E CÂNDIDO, 2014).

Basicamente, os indicadores ambientais podem ser definidos como uma forma de mensurar ou avaliar as pressões sobre o ambiente e sua situação, bem como demonstrar o nível de efetividade da gestão ambiental. Esses indicadores são transversais, podendo representar informações sobre o nível de preservação de áreas protegidas, condições da biodiversidade e qualidade de vida da população (FREITAS et al., 2011).

A utilização de indicadores, objetiva sistematizar um sistema de informações em uma base de dados realística, ao passo que os índices buscam representar e mensurar fenômenos e fatos complexos por meio de um número que sintetize a realidade, como se observa no Índice de Desenvolvimento Humano, no Índice de Performance Ambiental, no Índice de Vulnerabilidade Ambiental, entre outros (SCANDAR NETO, 2006). De acordo com Wilkie et al. (2006), corrobora para essa realidade a grande diversidade de indicadores ${ }^{4}$ e índices ${ }^{5}$, o que nos leva a refletir sobre a impossibilidade de se vislumbrar formas de mensurar a efetividade das APs que possam vir a ter ampla aceitação, mesmo com as limitações relacionadas à incipiência dos dados, além das discordâncias metodológicas e conceituais. Para essa questão, Januzzi (2006) chama a atenção para possíveis perdas conceituais no processo de aglutinação dos indicadores.

Sob essa perspectiva, Abramovay (2010) argumenta que os indicadores e índices possuem importante papel, por serem capazes de mensurar com eficácia a adoção de políticas públicas relevantes para a sociedade. Entretanto, deve-se considerar que os indicadores possuem a tendência de privilegiar evidências mais próximas e isoladas de acordo com a sua disponibilidade, limitando a análise e a compreensão das singularidades e complexidades dos fenômenos, além de possibilitar a construção de cenários que podem não ser realísticos (FRANCO NETTO E MIRANDA, 2011).

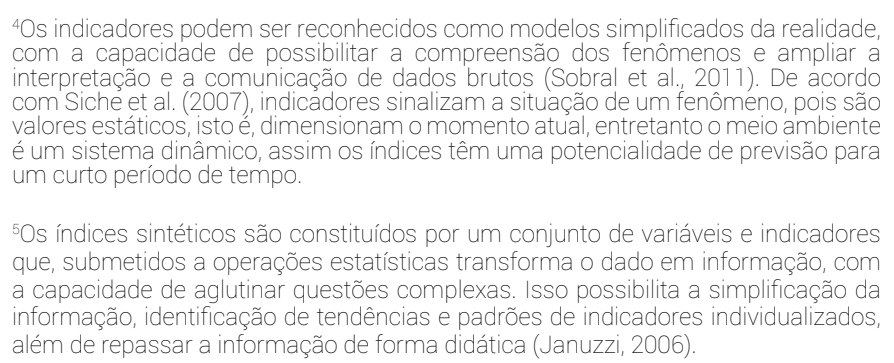

Para se ampliar a eficácia metodológica, Jannuzzi (2006) afirma que é preciso selecionar as variáveis de acordo com sua disponibilidade e relevância da temática analisada, pela confiabilidade dos dados, pelo grau de cobertura e por sua periodicidade de atualização. No entanto, em situações práticas, a disponibilidade dos dados acaba por ser condição sine qua non para construção dos indicadores e índices.

A seleção de indicadores não é um simples processo, tendo em vista que para a agregação de indicadores e posterior construção de um índice deve-se observar o modelo conceitual, além da relação de contiguidade e evolução dos fenômenos (SCANDAR NETO, 2006). A ponderação e a agregação de índices são as principais estratégias para sintetizar indicadores que de forma isolada seriam de difícil correlação, facilitando o repasse da informação para não especialistas, a partir da utilização de técnicas como a média aritmética, análise multivariada de componentes principais, a Análise de Processo Hierárquico - AHP, entre outros (JANNUZZI, 2006; HOCKINGS, 2006).

As medidas sintéticas podem contribuir em situações em que o analista ou gestor encontra-se incapacitado de analisar o significado de diagnósticos com um grande número de indicadores, tabelas, gráficos, imagens e mapas. Ressalta-se que a sintetização dos indicadores, apesar de ampliar a agregação das variáveis e simplificar as correlações dos dados, aumenta a generalização e a subjetividade das informações (JANNUZZI, 2006). Isso reduz a capacidade de se observar as singularidades dos fenômenos e diminui a proximidade com a realidade.

Mesmo com a consolidação de metodologias para mensuração e representação da realidade, observamse diversas limitações como a seleção de variáveis de baixa representatividade e confiabilidade, determinação de medidas e pesos aleatórios, com ausência de parâmetros estatísticos capazes de quantificar o nível de significância do indicador. Tais limitações trazem à tona fragilidades conceituais e metodológicas que acabam por servir a agentes políticos e empresariais no fomento e no repasse de informações, servindo a interesses específicos (CANAL et al, 2013).

O grande desafio da construção de indicadores é fornecer um retrato realístico de forma simples, apesar da incerteza e da complexidade. Nesse contexto, amplia-se a importância da correlação e da análise sistêmica das singularidades existentes em escalas local, regional e global em relação à diversidade ambiental e cultural. Isso ressalta os diferentes graus de desenvolvimento como importantes fatores na construção e na atualização dos indicadores e da adaptação de experiências exitosas no desenvolvimento de metodologias para avaliação da efetividade de APs (DAHL, 1997). 
Metodologias de avaliação da efetividade das áreas protegidas: aplicações e desafios

O V Congresso Mundial de Parques (DURBAN, 2003), preocupou-se em identificar lições aprendidas no processo de avaliação da efetividade de APs nas últimas décadas, definindo os temas que merecem maior atenção dos pesquisadores e gestores, como identificar e desenvolver metodologias e indicadores que representem a realidade e a dinâmica local (SILVA, 2016).

$\mathrm{Na}$ última década, dados de eficácia da gestão das APs foram coletados em 169 países, usando 69 metodologias diferentes que resultaram na formação do Banco de Dados Global sobre Eficácia do Gerenciamento de Áreas Protegidas (GD-PAME). De acordo com o relátorio da UNEP; WCMC; IUCN; NGS (2018), atualmente cerca de 9\% (21.743) das APs do planeta passaram por um processo de avaliação de efetividade. Isso corresponde a $19,9 \%$ da cobertura de territórios reconhecidos como protegidos no WDPA.

Considerando o quantitativo de APs avaliadas, o maior número é encontrado no continente europeu, alcançando $74 \%$ das avaliações mundiais. Ressalta-se que na Europa muitas APs possuem tamanhos reduzidos, e muitas destas foram repetidamente avaliadas, o que eleva o número de avaliações relatadas (UNEP; WCMC; IUCN; NGS, 2018) (Figura 2).

Somente a África e a América do Norte possuem mais de $30 \%$ da área total de sua rede de APs avaliada, apenas $21 \%$ dos países cumprem a meta de eficácia da gestão em terra e $16 \%$ dos países atingem a meta no oceano, segundo os dados da GD-PAME (Figura 3 e 4).

Figura 2 - Percentual de áreas protegidas avaliadas quanto ao nível de efetividade.

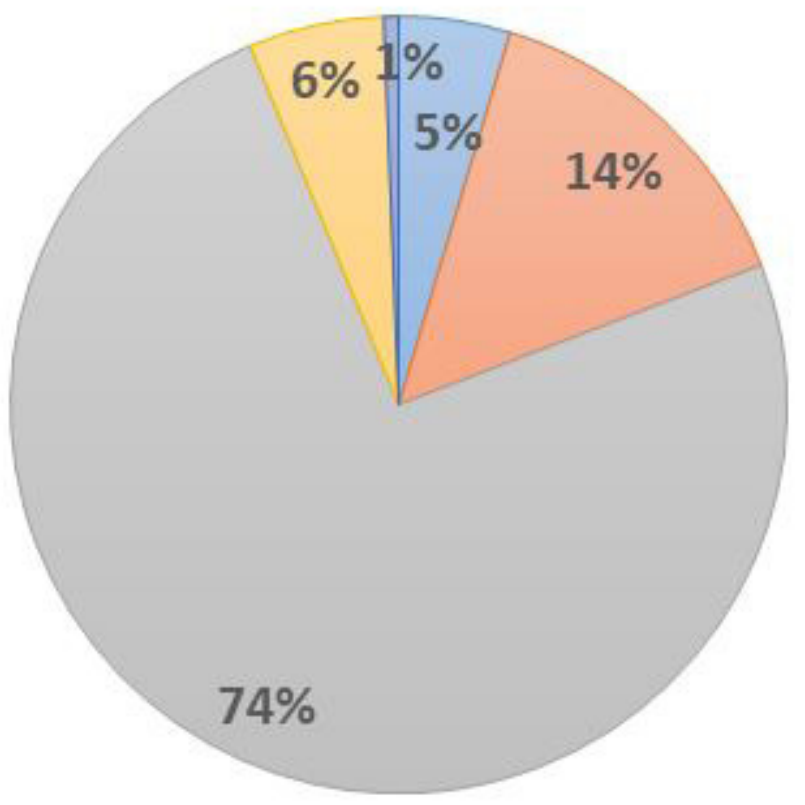


Figura 3 - Percentual de cobertura das áreas protegidas avaliadas quanto à efetividade por continente.

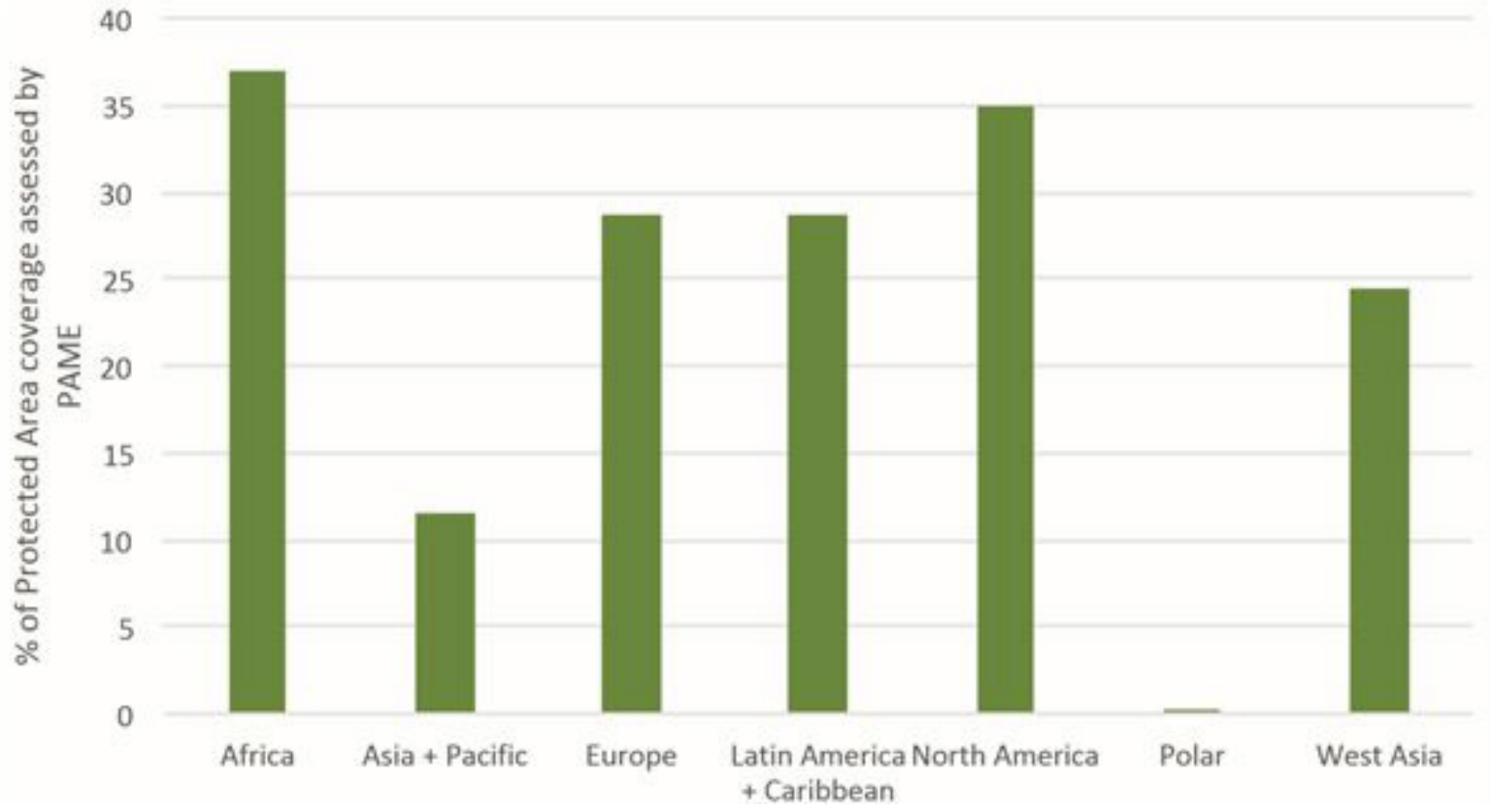

Fonte: UNEP-WCMC e IUCN, 2018.

Figura 4 - Percentual de cobertura das áreas protegidas avaliadas quanto à efetividade por país.

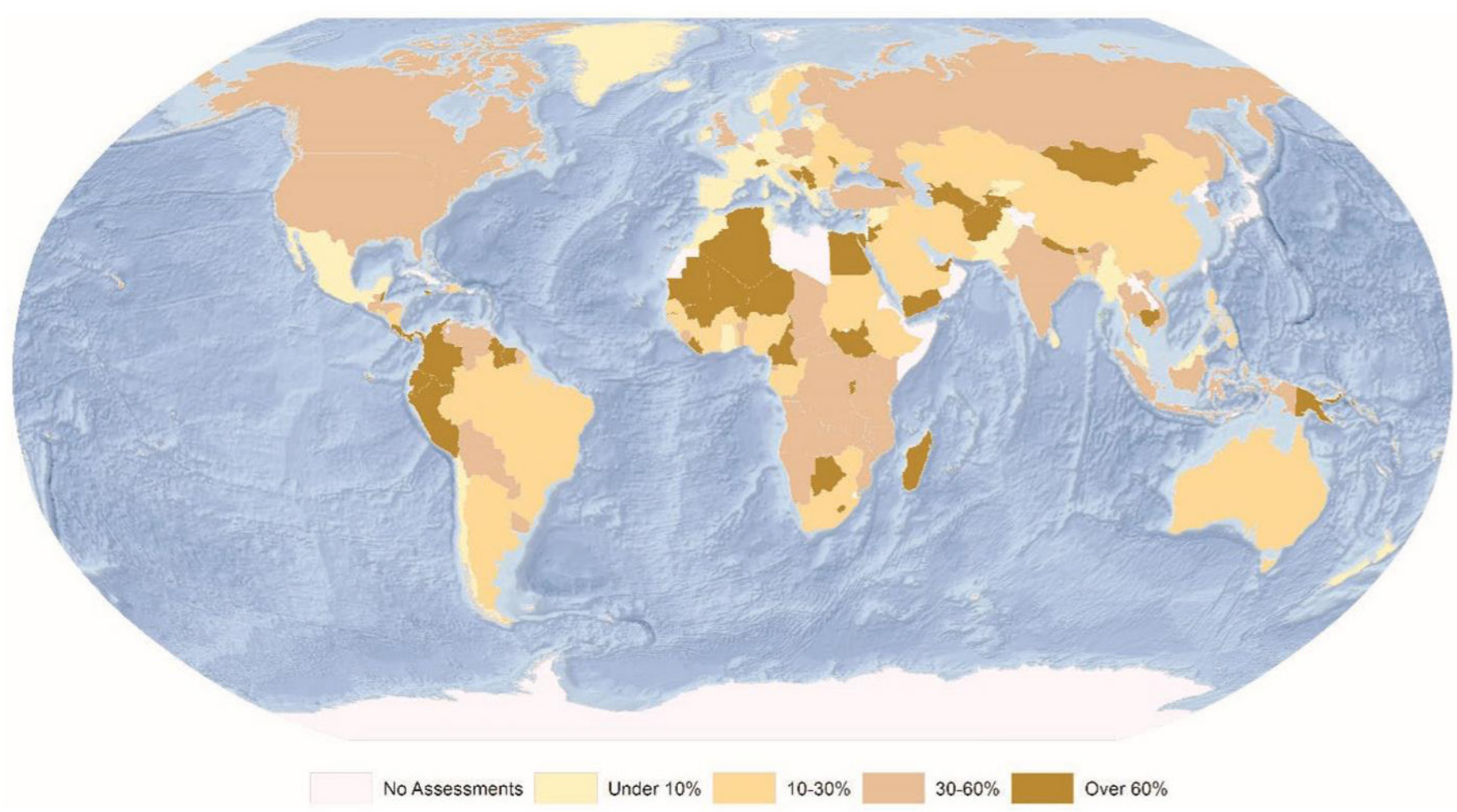

Fonte: UNEP-WCMC e IUCN, 2018. 
Conforme estudo desenvolvido pela UNEP-WCMC e a IUCN (2018), ao considerarmos o percentual de APs com gestão avaliadas como "eficazes" ou "altamente eficazes" o percentual reduziu de 54\% em 2014 para $48 \%$ em 2017. No entanto, a Perspectiva do Patrimônio Mundial da IUCN identifica que $64 \%$ das APs têm uma perspectiva de conservação positiva ("Bom" ou "Bom com algumas preocupações").

A partir da visão geral, verifica-se o desenvolvimento de diferentes metodologias na África, na Ásia, na América e na Europa. Mesmo com essas diversas metodologias desenvolvidas, Hockings et al. (2015) afirmam que ainda são poucos os países ou agências que estão avaliando o nível de efetividade das APs com periodicidade regular.
Um dos pressupostos da ciência é a refutação de hipóteses e teorias (MORIN et al., 2003). Nesse sentido, métodos com foco na análise de padrões da natureza ou como manejar a biodiversidade são constantemente reavaliados ou mesmo complementados. Percebese que os modelos produzidos até o momento devem ser aprimorados e revistos na tentativa de ampliar a aproximação com a realidade (PADUA E CHIARAVALLOTI, 2012). Seguindo esses princípios, diversas experiências no Brasil e na América Latina foram direcionadas para a construção e a consolidação de metodologias que possibilitassem a mensuração e a representação da efetividade das APs em escalas nacional, regional e local (Quadro 3).

Quadro 3 - Metodologias utilizadas no Brasil para avaliação da efetividade de unidades de conservação.

\begin{tabular}{|c|c|c|}
\hline METODOLOGIA & ORGANIZAÇÁO & PERIODO DE APLICAÇÁOO \\
\hline $\begin{array}{l}\text { Implementação e Vulnerabilidade de } \\
\text { Unidades de Conservação }\end{array}$ & WWF Brasil & 1999 \\
\hline $\begin{array}{c}\text { Certificação de Unidades de Conservação - } \\
\text { Parâmetros e procedimentos para } \\
\text { certificação do manejo de unidades de } \\
\text { conservação }\end{array}$ & $\begin{array}{l}\text { Instituto de Pesquisas } \\
\text { da Mata Atlântica - } \\
\text { IPEMA }\end{array}$ & 2002 \\
\hline Scenery Matrix & $\begin{array}{l}\text { Instituto Florestal do } \\
\text { Estado de São Paulo }\end{array}$ & $1999-2005$ \\
\hline Parkswatch & $\begin{array}{c}\text { Centro de } \\
\text { Conservação Tropical } \\
\text { da Universidade de } \\
\text { Duke e Fundação } 0 \\
\text { Boticário }\end{array}$ & $2002-2006$ \\
\hline $\begin{array}{l}\text { Indicadores da Efetividade de } \\
\text { Implementação (IEI) das UCs estaduais }\end{array}$ & SDS, Amazonas & 2006 \\
\hline $\begin{array}{l}\text { Programa de Monitoramento da } \\
\text { Biodiversidade e do Uso de Recursos } \\
\text { Naturais em Unidades de Conservação } \\
\text { Estaduais do Amazonas (PROBUC) }\end{array}$ & SDS, Amazonas & 2006 \\
\hline $\begin{array}{l}\text { iSAM - Indicadores Socioambientais de } \\
\text { Monitoramento }\end{array}$ & Siani et al. (2017) & $2000-2010$ \\
\hline $\begin{array}{l}\text { Protocolo de avaliação de efetividade de } \\
\text { gestão de mosaicos de áreas protegidas no } \\
\text { Brasil }\end{array}$ & Gidsicki, D. (2013) & 2013 \\
\hline RAPPAM & WWF Brasil e ICMBIO & $2005-2010-2015$ \\
\hline
\end{tabular}




\begin{tabular}{|c|c|c|}
\hline $\begin{array}{c}\text { FAUC - Ferramenta de Avaliação da } \\
\text { Efetividade do Programa Áreas Protegidas } \\
\text { da Amazônia }\end{array}$ & $\begin{array}{c}\text { Programa áreas } \\
\text { Protegidas da } \\
\text { Amazônia - Arpa }\end{array}$ & $2006-2015$ \\
\hline METT & Banco Munidal e WWF & $2005-2006$ \\
SISUC & $\begin{array}{c}\text { Instituto } \\
\text { Socioambiental }\end{array}$ & $2010-2015$ \\
\hline Avaliação de Mosaico & WWF & 2011 \\
\hline SAMGe & ICMBIO/WWF Brasil & $2015-2016$ \\
\hline Indice de Controle Ambiental - ICA & Masullo et al. (2018) & 2010 \\
\hline
\end{tabular}

Fonte: adaptado de Silva, 2016; WWF, 2017; Masullo et al., 2018a.

Segundo Silva (2016), essas metodologias devem utilizar modelos empíricos robustos, com um conjunto amplo de dados para estimar os efeitos que as UCs possuem sobre os resultados ambientais e sociais. Essa diferença possibilita alcançar, segundo a autora, a construção de um sistema efetivamente representativo e manejado, além de apresentar uma forma de se verificar que o investimento de tempo e esforço na criação e na gestão desses territórios resulta nos benefícios que a sociedade busca. A autora ressalta que, para o desenvolvimento de metodologias para a avaliação da efetividade de UCs, o marco conceitual deve ser utilizado como ponto de partida para adaptação das metodologias existentes, combinando diferentes procedimentos e abordagens.

Avanços, contribuições e desafios dos métodos de efetividade das áreas protegidas

Mesmo com os avanços metodológicos na última década apresentados, o percentual de APs avaliadas é extremamente reduzido (JUFFE-BIGNOLI et al., 2014). Segundo os autores, isso pode demonstrar descaso do poder público ao planejamento desses territórios e/ou mesmo que essas metodologias estão desconectadas com a realidade.

Em consonância com Leverington et al. (2010), StollKleemann (2010), Nelson e Chomitz (2011), Geldmann et al. (2014) e Silva (2016), observa-se a partir da avaliação metodológica desenvolvida que essas ferramentas diferem entre si, em relação à abordagem conceitual, aos objetivos e à forma de aplicação, à área de abrangência, aos indicadores, aos critérios de análise e aos softwares utilizados para a sintetização.

Especificamente em relação aos principais métodos aplicados no Brasil, observa-se que em geral são avaliações baseadas principalmente em dados primários de alta subjetividade, coletados por meio de questionários aplicados por meio eletrônico ou por workshops, porém com relativa participação dos gestores e comunidade local na construção dos indicadores. Esses métodos também apresentam dificuldade em estabelecer correlação entre os indicadores mensuráveis e os não mensuráveis. Outra questão a considerar é a disponibilidade de recursos financeiros, que influencia diretamente a qualidade dos resultados para avaliação, devido à natureza altamente flexível na implementação das metodologias (STOLLKLEEMANN, 2010).

De acordo com Siani et al. (2017), os métodos sistemáticos de monitoramento de UCs mais utilizados no Brasil em geral são voltados para biodiversidade, recursos naturais ou para avaliação e monitoramento da efetividade de gestão, com ênfase na avaliação do papel das instituições públicas no controle do SNUC. Para Marinelli (2011) essas metodologias utilizadas ainda relutam em considerar como a sociedade e suas múltiplas dimensões são afetadas ou beneficiadas pela criação e a gestão das UCs.

No entanto, os métodos analisados apresentamse como importantes ferramentas para a avaliação da efetividade de gestão das UCs do País, haja vista que as metodologias aplicadas revelam-se de maneiras diferenciadas, por se tratar de abordagens em níveis diversos. Segundo Júnior e Agra Filho (2015), a 
situação atual revela a necessidade de sua aplicação de modo complementar entre si e de outras abordagens metodológicas, que sejam mais fluidas e adaptativas com o objetivo de otimizar o planejamento e a gestão das UCs.

O envolvimento e as contribuições de vários grupos de partes interessadas podem ser cruciais. Os resultados da avaliação precisam ser considerados e integrados no processo de gestão, para tornar as APs mais eficientes (STOLL-KLEEMANN, 2010). Em meio ao desenvolvimento de técnicas e métodos apresentados, verifica-se que os avanços metodológicos observados buscam a integração dos processos de planejamento, gestão e a dinâmica territorial desses territórios protegidos, bem como trazem à tona não só resultados práticos, mas também novas abordagens de ação.

Entre os métodos ligados a avaliação da efetividade das UCs no Brasil, identifica-se um conjunto comum de indicadores com diferentes tipos de governança e níveis de proteção. Observa-se a interação entre estes elementos, que se conectam através de diferentes dimensões, representadas por indicadores selecionados.

Visualiza-se nitidamente que esforços foram sistematizados para simplificar a coleta de dados e a produção de relatórios, mas os custos elevados, além da falta de dados abrangentes e consistentes, tornaram surpreendentemente difícil avaliar efetividade dessas áreas protegidas. Nesse contexto, destacam-se as UCs estaduais e municipais, com estruturas precárias de planejamento e gestão. Isso reduz a capacidade de coleta, gerenciamento e monitoramento de indicadores, dificultando inclusive a avaliação da efetividade das UCs em escala nacional.

Para Campedelli et al. (2010), nesse contexto o desenvolvimento de metodologias associadas às tecnologias e as diferentes realidades territoriais das APs tornam possível a busca constante por melhores soluções aos desafios interligados a esses territórios. Possibilita-se, dessa forma, a redução da subjetividade, além de determinar a capacidade de uma metodologia em fornecer as respostas certas a uma dada questão. Entretanto, a simples avaliação da efetividade através da aplicação de indicadores e índices, embora necessária, poderá não ser suficiente para mensurar os objetivos da AP (HOCKINGS et al., 2015).

Diante desses métodos e pressupostos científicos, observa-se a necessidade do desenvolvimento de análises mais precisas sobre a realidade, considerando que a construção de novos métodos em escalas regional e local permitem um menor esforço físico/ monetário e com resultados mais precisos (PADUA E CHIARAVALLOTI, 2012).

\section{Conclusão}

No presente trabalho, foi possível observar que, mesmo com as mais diversas limitações metodológicas e conceituais, o uso de indicadores e índices para avaliação e planejamento de políticas públicas vem passando por avanços e pode possibilitar importantes subsídios e efeitos positivos para a otimização do planejamento e da gestão de políticas em escalas global, regional e local.

Em linhas gerais, a construção das metodologias analisadas possui o objetivo de estruturar sistemas que possibilitem a articulação do monitoramento, levando em consideração determinantes e condicionantes ambientais, socioeconômicos e culturais. 0 intuito é representar a realidade de forma sintética, em diferentes níveis e dimensões.

Entretanto, deve-se entender que o efeito das APs sobre os valores de conservação só pode ser avaliado ao longo do tempo, com o entendimento de que a efetividade da gestão desses territórios está intrinsecamente relacionada ao planejamento, à gestão e à resiliência dessas áreas. Ao mesmo tempo, verifica-se que os indicadores devem ser analisados de forma sistêmica, entendendo suas inter-relações e limitações estatísticas que surgem das complexidades e singularidades territoriais.

Limitações como a escassez de informações e a descontinuidade na produção de dados estatísticos não permitem comparar o desempenho alcançado pelas APs ao longo do tempo. Somado a isso, nota-se grande subjetividade na seleção e na ponderação dos indicadores. Outro fator a se destacar é o possível uso restrito dos indicadores em trabalhos acadêmicos e científicos sem o conhecimento dos gestores públicos ou da população, subutilizando as metodologias desenvolvidas, ou mesmo possibilitando seu uso para interesses políticos (MORAES et al., 2016).

Como indicação de pesquisa futura, sugere-se um estudo detalhado, visando à criação de um método com capacidade de mensurar os pontos carentes de ações e medidas necessárias à efetiva governança institucional, social, econômica e ambiental, de forma a subsidiar a adoção de políticas públicas efetivas. 


\section{Referências}

ABRAMOVAY, R. (2010). Desenvolvimento sustentável: qual a estratégia para o Brasil? Novos Estudos, 87, p. 97-113.

BERTZKY, B. et al. (2012). Protected Planet Report 2012: Tracking progress towards global targets for protected areas. IUCN, Gland, Switzerland and UNEP-WCMC, Cambridge, UK, 68p.

CAMPEDELLI, T. et al. (2010). Effectiveness of the Italian national protected areas system in conservation of farmland birds: a gap analysis. Ardeola, 57, p. 51-64.

CANAL, F. V.; BRASIL, G. H.; GARCIA, E. C. (2013). Construção de um indicador sintético-sistêmico de desenvolvimento sustentável: municípios do Estado do Espírito Santo. GEPROS. Gestão da Produção, Operações e Sistemas, Bauru, Ano 8, n. 1, p. 133-146.

CASTRO JÚNIOR, E.; COUTINHO, B. H.; FREITAS, L. E. (2009). Gestão da Biodiversidade e áreas protegidas. In: GUERRA, A. J e COELHO, M. C. N. (orgs). Unidades de Conservação: abordagens e características geográficas. Bertrand Brasil. Rio de Janeiro. p. $25-66$.

CIFUENTES, M. et al. (2000). Medición de la efectividad del manejo de áreas protegidas. WWF: IUCN: GTZ, Turrialba, $105 p$.

COAD, L. et al (2015). Measuring impact of protected area management interventions: Current and future use of the global database of protected area management effectiveness. Phil. Trans. R. Soc. B 370.

DAHL, A. L. (1997). The big picture: comprehensive approaches. In: MOLDAN G.; BILHARZ, S. (eds.) Sustainability indicators: report of the project on indicators of sustainable development. Chichester: John Wiley \& Sons Ltd., p. 69-83.

DIEGUES, A. C. S. (2000). O mito moderno da natureza intocada. Núcleo de Apoio à Pesquisa sobre Populações Humanas e Áreas Úmidas Brasileiras 3. ed. São Paulo: Hucitec, USP, 161p.

DUDLEY, N.; STOLTON, S. (2003). Running Pure: he Importance of Forest Protected Areas to Drinking Water. World Bank/WWF Alliance for Forest Conservation and Sustainable Use. WWF, Gland, Switzerland. Earth Trends.

DUDLEY, N. (2008). Directrices para la aplicación de lãs categorías de gestión de áreas protegidas. UICN, Gland. (Suiça). 96p.

ERVIN, J. (2003). WWF: Rapid Assessment and Prioritization of Protected Area Management (RAPPAM) Methodology. WWF, Gland, Switzerland, $52 \mathrm{p}$.

FRANCO NETTO, G.; MIRANDA, A. C. (2011). Saúde Ambiental: guia básico para a construção de indicadores. In: BRASIL, Ministério da Saúde. Secretaria de Vigilância em Saúde. Saúde Ambiental: guia básico para a construção de indicadores. Ministério da Saúde. Brasília, p. 13-17.

FRANKS, P.; SCHRECKENBERG K. (2016). Advancing Equity in Protected Area Conservation. The International Institute for Environment and Development (IIED).

FREITAS. C. M. (2011). Construindo indicadores em saúde ambiental. In: BRASIL, Ministério da Saúde. Secretaria de Vigilância em Saúde. Saúde Ambiental: guia básico para a construção de indicadores. Ministério da Saúde. Brasília, p. 19-24.

GELDMANN, J. et al. (2014). Mapping change in human pressure globally on land and within protected areas. Conservation Biology, 28, p. 1.604-1.616.

(2015). Changes in Protected Area Management Effectiveness over Time: A Global Analysis. Biological Conservation, 191. Elsevier B. V., p. 692-699.

(2017). A global analysis of management capacity and ecological outcomes in terrestrial protected areas. Conservation Letters, p. 1-10. 
GETZNER, M.; JUNGMEIER, M.; PFLEGER. B. (2012). Evaluating Management Effectiveness of National Parks as a Contribution to Good Governance and Social Learning. InTech/Open Science. Protected Area Management, p. 129146.

GIDSICKI, D. (2013). Protocolo de avaliação de efetividade de gestão de mosaicos de áreas protegidas no Brasil. Dissertação de Mestrado Profissionalizante em Gestão de Áreas Protegidas na Amazônia. Instituto Nacional de Pesquisas da Amazônia. Programa de Pós-Graduação do INPA. São Paulo, 91 p.

GRAEME, S. et al. (2015). Understanding protected area resilience: a multi-scale, social-ecological approach. Ecological Applications, 25(2). p. 299-319.

HIRSCHNITZ-GARBERS M.; STOLL-KLEEMANN, S. (2011). Opportunities and barriers in the implementation of protected area management: A qualitative meta-analysis of case studies from European protected areas. The Geographical Journal. 177 (4).. p. 321-334.

HOCKINGS, M. et al. (2004). Management effectiveness: assessing management of protected areas? Journal of Environmental Policy and Planning 6. p. 157-174.

HOCKINGS, M.; STOLTON, S.; LEVERINGTON, F.; DUDLEY, N.; COURRAU, J. (2006). Evaluating Effectiveness: A framework for assessing management effectiveness of protected areas. IUCN, Gland, Suíça, Cambridge, Reino Unido.

HOCKINGS, M. et al. (2015). Protected area management effectiveness. In: WORBOYS, G. L.; LOCKWOOD, M.; KOTHARI, A.; FEARY, S.; PULSFORD, I. (eds.). Protected Area Governance and Management. Canberra: ANU Press, p. 889-928.

JANNUZZI, P. M. (2006). Indicadores sociais no Brasil. Campinas: Alínea, 141p.

JENKINS, C. N.; JOPPA, L. (2009). Expansion of the global terrestrial protected area system. Biological Conservation, 142 , p. 2.166-2.174.

JUFFE-BIGNOLI, D. et al. (2014). Protected Planet Report 2014: Tracking progress towards global targets for protected areas. UNEP-WCMC, Cambridge, UK.

JÚNIOR, L. C. de A.; AGRA FILHO, S. S. (2015). Estudo comparativo entre três diferentes métodos de avaliação da efetividade de gestão de áreas protegidas. Revista GESTA, v. 3, n. 1, p. 232-241.

LEVERINGTON, F. et al. (2010). A Global Analysis of Protected Area Management Effectiveness. Environmental Management.

MAIOR, M. M. S; CÂNDIDO, G. A. (2014). Avaliação das metodologias brasileiras de vulnerabilidade socioambiental como decorrência da problemática urbana no Brasil. Cad. Metrop., São Paulo, v. 16, n. 31, p. 241-264.

MARINELLI, C. E. (2011). De olho nas unidades de conservação: Sistema de Indicadores Socioambientais para Unidades de Conservação da Amazônia Brasileira. Instituto Socioambiental. São Paulo, 12. p.

MASULLO, Y. A. M. et al. (2018a). Índice de Controle Ambiental das Unidades de Conservação do Maranhão. Revista do Departamento de Geografia (USP), v. 36. p. 104-116.

(2018b). Socioeconomic dynamics of the protected areas of Maranhão. CONFINS (Paris), v. 38, p. 1-13.

MCNEELY, J. A. (org.). (1993). Parks for Life: Report of the 4th World Congress on National Parks and Protected Areas. IUCN, Gland, Switzerland.

(2004). At least do no harm: poverty and protected areas in China. Discussion paper for the CCICED. Protected Areas Task Force.

MILANO, M. S. (2012). Um olhar para além da Amazônia Brasileira. In: FUNDO VALE. Áreas protegidas. Rio de Janeiro: Fundo Vale, p. 14-39.

MORAES, D. E. et al. (2016). Indicadores sintéticos de qualidade de vida e o conceito de natureza/ambiente. Caminhos 
de Geografia Uberlândia, v. 17, n. 58, p. 123-135.

MORI, K.; CHRISTODOULOU, A. (2012). Review of sustainability indices and indicators: Towards a new City Sustainability Index (CSI). Environmental Impact Assessment Review 32, p. 94-106.

MORIN, E. et al. (2003). Educar na era planetária: o pensamento complexo como método de aprendizagem pelo erro e incerteza humana. Trad. Sandra Trabucco Mayra Valenzuela. São Paulo: Cortez; Brasília; UNESCO. 111 p.

NELSON, A. K.; CHOMITZ, M. (2011). Effectiveness of Strict vs. Multiple Use Protected Areas in Reducing Tropical Forest Fires: A Global Analysis Using Matching Methods. PLOS ONE, Volume 6, Issue 8, p. 01-14.

NOLTE, C. et al. (2013). Governance Regime and Location Influence Avoided Deforestation Success of Protected Areas in the Brazilian Amazon. Proceedings of the National Academy of Sciences 110 (13). p. 4.956-4.961.

PADOVAN, M. da P. (2003). Certificação de unidades de conservação. Conselho Nacional da Reserva da Biosfera da Mata Atlântica. São Paulo, 124 p.

(2001). Formulación de un estandar y un procedimiento para la certificación del manejo de areas protegidas. Centro Agronómico Tropical de Investigación y Enseñanza. Turrialba, Costa Rica.

PADUA, C. V.; CHIARAVALLOTI, R. M. (2012). Pesquisa e conhecimento na gestão de unidades de conservação. In: CASES, M. O. (org.). Gestão de Unidades de Conservação: compartilhando uma experiência de capacitação. WWFBrasil/IPÊ - Instituto de Pesquisas Ecológicas. WWF-Brasil, Brasília, p. 139-153.

SALAFSKY, N. et al. (2008). A Standard Lexicon for Biodiversity Conservation: Unified Classifications of Threats and Actions. Conservation Biology, v. 22, p. 897-911.

SCANDAR NETO, W. J. (2006). Síntese que organiza o olhar: uma proposta para construção e representação de indicadores de desenvolvimento sustentável e sua aplicação para os municípios fluminenses. Dissertação de Mestrado, ENCE - Escola Nacional de Ciências Estatísticas, Rio de Janeiro, 106p.

SCHAEFER, M. et al. (2015). Nature as capital: advancing and incorporating ecosystem services in United States federal policies and programs. PNAS 112, p. 7.383-7.389.

SCHERL, L. M. et al. (2006). As áreas protegidas podem contribuir para a redução da pobreza? Oportunidades e limitações. IUCN, Gland, Suíça e Cambridge, Reino Unido, 60p.

SCHRECKENBER, K. et al. (2016). Unpacking Equity for Protected Area Conservation. PARKS, v. 22.2, p. 11-26.

SCHULZE, K. et al. (2017). Na assessment of threats to terrestrial protected áreas. Conservation Letters, p. 1-10.

Siani, S. M., Amaral, S., \& Monteiro, A. M. (2017). iSAM - Um sistema de indicadores para o monitoramento da Área de Proteção Ambiental Mananciais do Rio Paraíba do Sul. Revista Do Departamento De Geografia, 33, 63-73.

SICHE, R. et al. (2007). Índices versus indicadores: precisões conceituais na discussão da sustentabilidade de países. Ambiente \& Sociedade, v. 10, n. 2, p. 137-148.

SILVA, O. D. A. (2016). Estratégia do Programa Áreas Protegidas da Amazônia para avaliar a Efetividade das Unidades de Conservação. Tese de Doutorado. Universidade de Brasília - UnB, Centro de Desenvolvimento Sustentável - CDS, $212 \mathrm{p}$.

SOBRAL, A. et al. (2011). Definições básicas: dado, indicador e índice. In: BRASIL, Ministério da Saúde. Secretaria de Vigilância em Saúde. Saúde Ambiental: guia básico para a construção de indicadores. Ministério da Saúde. Brasília, p. 25-52.

SOUZA, L. B. (2015). A hipérbole mercantil da expansão urbana e suas implicações ambientais. Revista Mercator, v. 14, n. 4, Número Especial, Fortaleza, p. 159-180.

STOLL-KLEEMANN, S. (2010). Evaluation of management effectiveness in protected areas: Methodologies and results. 
Basic and Applied Ecology 11, p. 377-382.

STOLL-KLEEMANN, S.; JOB, H. (2008). The Relevance of Effective Protected Areas for Biodiversity Conservation: An Introduction. GAIA 17, p. 186-189.

STOLL-KLEEMANN, S.; O'RIORDAN, T. (2017). The challenges of the Anthropocene for biosphere reserves. PARKS, vol. 23, p. 89-100.

STOLTON, S. et al. Reporting Progress in Protected Areas a SiteLevel Management Effectiveness Tracking Tool: second edition. World Bank/WWF Forest Alliance published by WWF, Gland, Switzerland. 2007. 21p.

LEVERINGTON; F. (2007). Reporting Progress in Protected Areas a SiteLevel Management Effectiveness Tracking Tool: second edition. World Bank/WWF Forest Alliance published by WWF, Gland, Switzerland.

TERBORGH, J.; SCHAIK, C. V. (2002). Por que o mundo necessita de parques? In: Tornando os parques eficientes: estratégias para conservação da natureza nos trópicos. Curitiba: Universidade Federal do Paraná, p. 25-36.

UNEP-WCMC; IUCN; NGS (2018). Protected Planet Report 2018. UNEP-WCMC, IUCN and NGS: Cambridge UK; Gland, Switzerland; and Washington, D.C., USA, 70p.

VEIGA, J. E. da (2009). Indicadores socioambientais: evolução e perspectivas. Revista de Economia Política 29 (4), p. 421-435.

WATTS, J. E. M. (2016). Marxan.io user guide: A web app for systematic conservation planning. Australian Research Council Centre of Excellence for Environmental Decisions. The University of Queensland, 25p.

WILKIE, D. S. et al. (2006). Parks and people: assessing the human welfare effects of establishing protected areas for biodiversity conservation. Conservation Biology: the journal of the Society for Conservation Biology, v. 20, n. 1, p. 247249.

WWF - World Wide Found for Nature Brasil. (1999). Áreas Protegidas ou Espaços Ameaçados: O Grau de Implementação e a Vulnerabilidade das Unidades de Conservação Federais Brasileiras de Uso Indireto. Orgs.: SÁ, Rosa M. Lemos de; FERREIRA, Leandro. Brasília, p. 33.

WWF Brasil - World Wide Found for Nature. (2017). Avaliação da gestão das unidades de conservação: métodos RAPPAM (2015) e SAMGE (2016). WWF Brasil. $1^{\text {a }}$ ED. Brasília. 127p. 